\title{
Intraosseous hemangioma of the orbit
}

\author{
June Seok Choi ${ }^{1}$, \\ Yong Chan $\mathrm{Bae}^{1,2}$, \\ Gyu Bin Kang ${ }^{1}$, \\ Kyung-Un Choi ${ }^{3}$ \\ ${ }^{1}$ Department of Plastic and Reconstructive \\ Surgery, Pusan National University School of \\ Medicine, Busan; \\ ${ }^{2}$ Biomedical Research Institute, Pusan \\ National University Hospital, Busan; \\ ${ }^{3}$ Department of Pathology, Pusan National \\ University School of Medicine, Busan, Korea
}

\begin{abstract}
Intraosseous hemangioma is an extremely rare tumor that accounts for $1 \%$ or fewer of all osseous tumors. The most common sites of its occurrence are the vertebral column and calvaria. Occurrence in a facial bone is very rare. The authors aim to report a case of the surgical treatment of intraosseous hemangioma occurring in the periorbital region, which is a very rare site of occurrence and to introduce our own experiences with the diagnosis and treatment of this condition along with a literature review. A 73-year-old male patient visited our hospital with the chief complaint of a mass touching the left orbital rim. A biopsy was performed by applying a direct incision after local anesthesia. Eventually, intraosseous hemangioma was diagnosed histologically. To fully resect the mass, the orbital floor and zygoma were exposed through a subciliary incision under general anesthesia, and then the tumor was completely eliminated. Bony defect was reconstructed by performing a seventh rib bone graft. Follow-up observation has so far been conducted for 10 months after surgery without recurrence or symptoms.
\end{abstract}

Keywords: Intraosseous / Hemangioma / Orbit

\section{INTRODUCTION}

Intraosseous hemangioma is an extremely rare tumor that accounts for $1 \%$ or fewer of all osseous tumors. Its peak incidence is in patients in their 40 s to $50 \mathrm{~s}$, and females are more commonly affected by the disease than males. The most common sites of its occurrence are the vertebral column and calvaria. Occurrence in a facial bone is very rare $[1,2]$.

It is usually indolent and grows slowly. Its radiological findings typically involve honeycomb or soap bubble shapes on computed tomography (CT) or magnetic resonance imaging (MRI). Moreover, it can present in a variety of forms. Caution should be paid when performing a biopsy because a massive hemorrhage can occur. When symptoms are present, it is important to perform surgery only after fully understanding the lesion, and the main treatment strategy is the surgical elimination of the tumor.

Correspondence: Yong Chan Bae

Department of Plastic and Reconstructive Surgery, Pusan National University School of Medicine and Biomedical Research Institute, Pusan National University Hospital, 179 Gudeok-ro, Seo-gu, Busan 49241, Korea

E-mail: baeyc2@hanmail.net

Received November 3, 2017 / Revised March 1, 2018 / Accepted March 9, 2018
The authors aim to report a case of the surgical treatment of intraosseous hemangioma occurring in the periorbital region, which is a very rare site of occurrence, and to introduce our own experiences with the diagnosis and treatment of this condition along with a literature review.

\section{CASE REPORT}

A 73-year-old male patient visited Pusan National University Hospital with the chief complaint of a mass touching the left orbital rim that had been present for a month. The patient did not complain of other symptoms such as tenderness and had no trauma history.

According the medical history of the patient, he had brow ptosis and lagophthalmos symptoms due to facial paralysis that occurred after otitis media surgery performed 30 years previously (Fig. 1). The CT findings showed a honeycombed osseous lesion in the left lateral orbital rim, and a benign bone tumor was suspected (Fig. 2). A biopsy was performed by applying a direct incision after local anesthesia of the lesion for a histological diagnosis, and bleeding continued for several days after the biopsy. The his-

68 Copyright $\odot 2018$ The Korean Cleft Palate-Craniofacial Association 


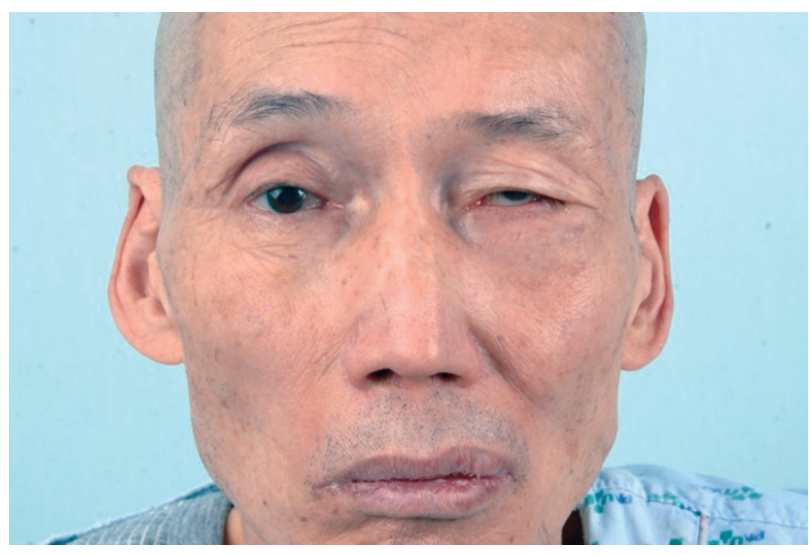

Fig. 1. A 73-year-old man with a slowly growing hard mass in the left infraorbital region.
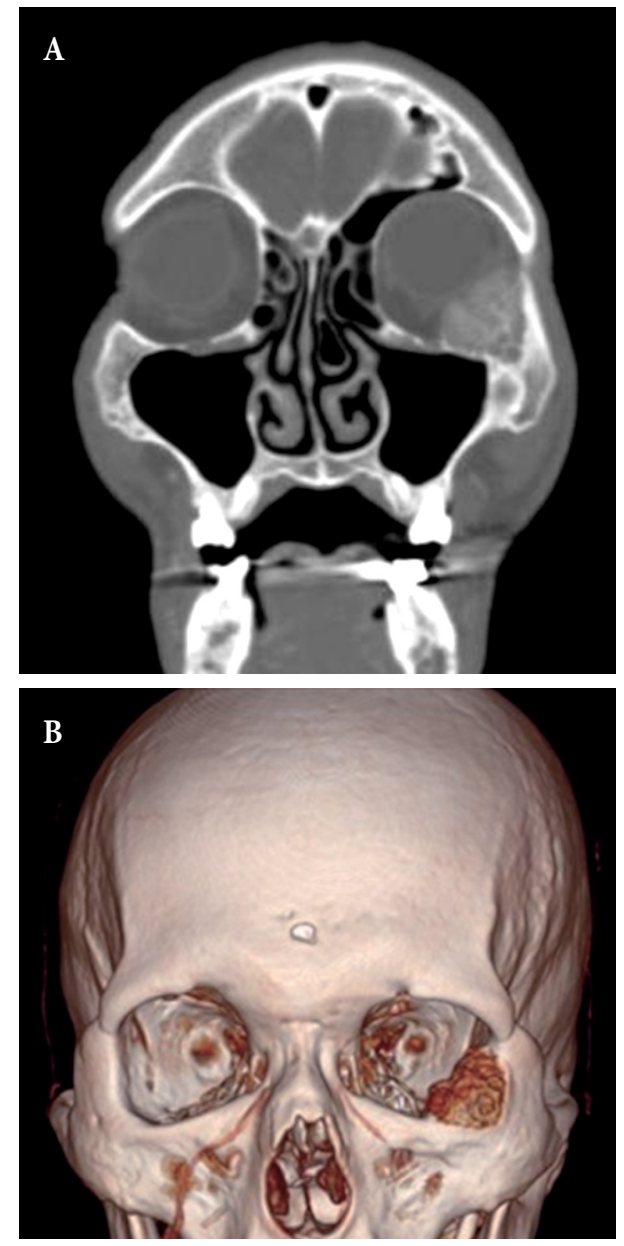

Fig. 2. Preoperative computed tomography images. A honeycombshaped bony lesion in the orbit (A: coronal, B: three dimension). tological examination showed multiple dilated vascular spaces between pre-existing bony trabeculae in a low-power view, and vascular spaces surrounded by endothelial cells in a high-power view. Eventually, intraosseous hemangioma was diagnosed histologically (Fig. 3).

To fully resect the mass, the orbital floor and zygoma were exposed through a subciliary incision under general anesthesia, and then the tumor was completely eliminated through sawing, curettage, and burring. Bleeding control was performed using electrocautery (Cutanplast, Mascia Brunelli, Milano, Italy and Surgicel, Ethicon, Somerville, NJ, USA) and the bony defect that occurred after the elimination of the tumor was reconstructed by perform-
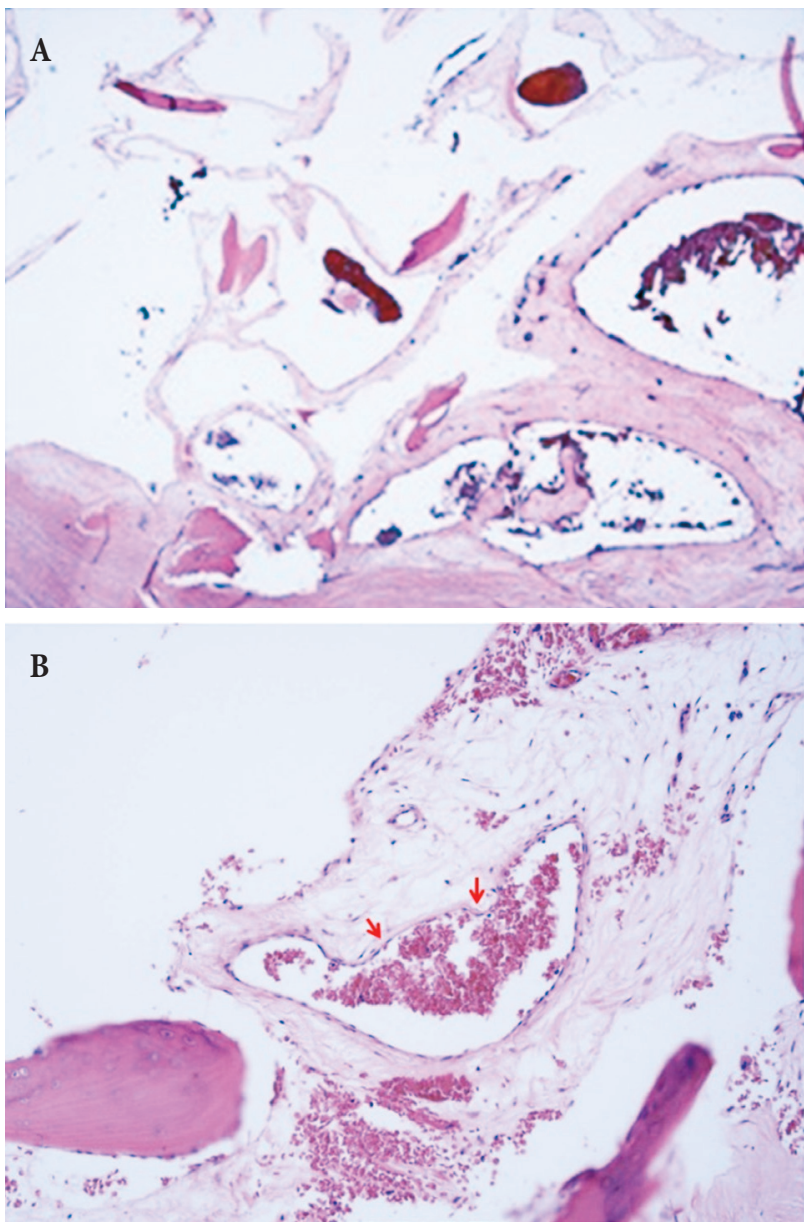

Fig. 3. Histopathologic findings. (A) In a low-power view, multiple dilated vascular spaces are present between pre-existing bony trabeculae $(\mathrm{H} \& \mathrm{E}, \times 40)$. (B) These vascular spaces are lined by flattened endothelial cells (arrows) $(\mathrm{H} \& \mathrm{E}, \times 200)$. 
ing a seventh rib bone graft. In the final histological diagnosis, the disease was confirmed as intraosseous hemangioma originating from the zygomatic bone, and follow-up observation has so far been conducted for 10 months after surgery without recurrence or symptoms (Fig. 4).

\section{DISCUSSION}

Intraosseous hemangioma is a slow-growing benign tumor of capillary, cavernous, and venous origin, and is also a rare tumor that accounts for $0.5 \%-1 \%$ of all osseous tumors. The most common sites of its occurrence are the calvaria and vertebral column. It is known that this tumor occurs more rarely in the facial bone although it occurs in other areas, such as the zygoma, orbit, ethmoid, and maxilla [3].

As a usually indolent tumor, it grows slowly and can cause facial deformation. When it develops in the orbit, it can be accompanied by exophthalmos, diplopia, and loss of sight. When the tumor invades the alveolar ridge, ulorrhagia can occur and teeth can be lost. Moreover, symptoms such as epistaxis, rhinanchone, diplopia, dysesthesia, exophthalmos, and rhinosinusitis can appear in such cases [4].

The diseases that must be differentiated from intraosseous hemangioma include exostosis, eosinophilic granuloma, fibrous dysplasia, multiple myeloma, dermoid cyst, and osteoid osteoma. The characteristic radiological findings of intraosseous hemangi- oma are osteolytic lesions with honeycomb, soap bubble, and radial shapes. The actual size of the lesion can be underestimated in plain radiographic examinations [5]. Therefore, CT is necessary for diagnosing this lesion. Some authors have claimed that MRI is very important when evaluating lesions abundant with blood vessels, such as intraosseous hemangioma. Because biopsy can cause severe bleeding, it is not necessarily needed if the radiological findings are typical. Otherwise, however, a biopsy should be performed with great caution.

It is known that the best treatment is surgical resection, and there should be no functional and aesthetic problems after resection. For intraosseous hemangioma with no or slight symptoms, surgical resection is not needed, and needle aspiration may be attempted for small intraosseous hemangiomas. Although radiotherapy or sclerotherapy was used in the past, radiotherapy can cause complications such as tissue necrosis, developmental disorders of the bones or teeth, radiation-induced sarcoma, and hemangioma caused by radiotherapy. Sclerotherapy is more useful for soft-tissue hemangioma. However, it can be used as an adjunct to surgery for intraosseous hemangiomas, and if it is performed within 48 hours before surgery, it can reduce the bleeding during surgery [2].

Small defects at the surgical site generated after the removal of the tumor can be reconstructed through a pedicled flap or skin graft, whereas large defects generally require facial bone, rib, or synthetic materials. When evaluating a facial mass, intraosseous
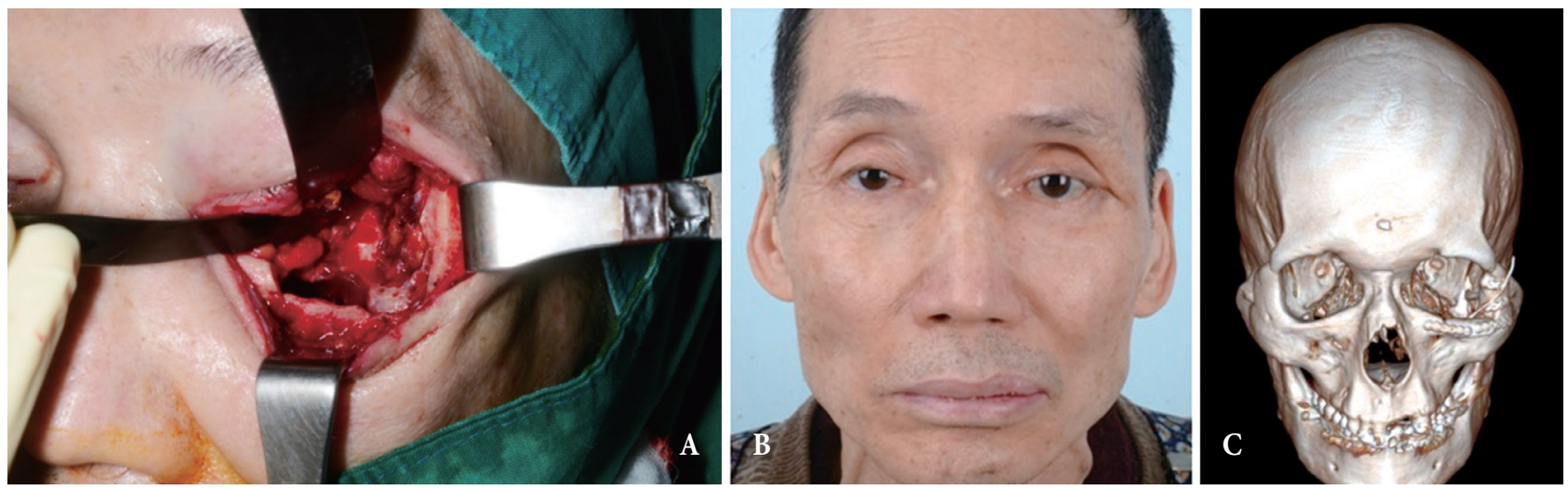

Fig. 4. Intraoperative and postoperative photos. (A) Intraoperative photo showing complete elimination through sawing, curettage, and burring. (B) Postoperative photo showing reconstruction by a seventh rib bone graft. (C) Postoperative photo 10 months after surgery. 
hemangioma must be included in the differential diagnosis, although it is very rare. First, the patient's history should be investigated thoroughly and a careful physical examination should be performed. If the typical findings of intraosseous hemangioma, such as soap-bubble shapes or radial osteolytic lesions, are observed in radiographic examinations such as CT or MRI, a biopsy is not mandatory. Otherwise, however, the biopsy must be performed very carefully. For treatment, complete resection is used principally if there are no functional or aesthetical problems, and sclerotherapy can be used as an adjunct to treatment [6].

The authors report this case and introduce our own experiences with the diagnosis and treatment of this condition, along with a literature review, because hemangiomas originating from the periorbital region are very rare and no previous domestic report has been published.

\section{CONFLICT OF INTEREST}

No potential conflict of interest relevant to this article was reported.

\section{PATIENT CONSENT}

The patients provided written informed consent for the publication and the use of their images.

\section{REFERENCES}

1. Madge SN, Simon S, Abidin Z, Ghabrial R, Davis G, McNab A, et al. Primary orbital intraosseous hemangioma. Ophthal Plast Reconstr Surg 2009;25:37-41.

2. Cheng NC, Lai DM, Hsie MH, Liao SL, Chen YB. Intraosseous hemangiomas of the facial bone. Plast Reconstr Surg 2006;117:2366-72.

3. Jung JH, Jeong BS, Kim ST, Kang IG. A case of intraosseous hemangioma of the maxilla bone. Korean J Otorhinolaryngol Head Neck Surg 2013;56:45-7.

4. Zou ZJ, Wu YT, Sun GX, Zhu XP, Meng XZ, He ZQ. Clinical application of angiography of oral and maxillofacial hemangiomas: clinical analysis of seventy cases. Oral Surg Oral Med Oral Pathol 1983;55:43747.

5. Sweet C, Silbergleit R, Mehta B. Primary intraosseous hemangioma of the orbit: CT and MR appearance. AJNR Am J Neuroradiol 1997;18:379-81.

6. Kaya B, Isilgan SE, Cerkez C, Otrakci V, Serel S. Intraosseous cavernous hemangioma: a rare presentation in maxilla. Eplasty 2014;14:e35. 